\title{
In-Place Query Driven Big Data Platform: Applications to Post Processing of Environmental Monitoring
}

\author{
Jen-Gaw Lee \\ National Center for High- \\ Performance Computing, NARL \\ 7, R\&D 6th Rd., Hsinchu Science \\ Park, Hsinchu 300, Taiwan \\ jglee0318@gmail.com
}

\author{
Ching-Yao Lin \\ National Center for High- \\ Performance Computing, NARL \\ 7, R\&D 6th Rd., Hsinchu Science \\ Park, Hsinchu 300, Taiwan \\ chingyao@nchc.narl.org.tw
}

\author{
Whey-Fone Tsai* \\ National Center for High- \\ Performance Computing, NARL \\ 7, R\&D 6th Rd., Hsinchu Science \\ Park, Hsinchu 300, Taiwan \\ wftsai@nchc.narl.org.tw
}

\author{
Hsi-Ching Lin \\ National Center for High- \\ Performance Computing, NARL \\ 7, R\&D 6th Rd., Hsinchu Science \\ Park, Hsinchu 300, Taiwan \\ sclin@nchc.narl.org.tw
}

\author{
Lung-Cheng Lee \\ National Center for High- \\ Performance Computing, NARL \\ 7, R\&D 6th Rd., Hsinchu Science \\ Park, Hsinchu 300, Taiwan \\ Iclee@nchc.narl.org.tw \\ Ben-Jei Tsuang \\ National Chung-Hsing University \\ 250 Kuo Kuang Rd., \\ Taichung 402, Taiwan
}

btsuang@gmail.com

\begin{abstract}
In this paper, we describe the work on the in-place query driven big data platform and applications built on the platform, which include processing climate simulation data and air pollution monitoring. The system architecture of this experimental platform comprises NCHC supercomputer, ALPS, storage pool, one master data node and 18 slave data nodes. The openSUSE operating system and MaraiaDB database are installed on all nodes. Master node is responsible for metadata management and information integration and 18 slave nodes for distributed database and parallel model simulation and computation/analysis. The data are distributed to local nodes according to a pre-defined data partition plan. When application software, such as simulation model or post-processing application, is executed on slave nodes, the relevant input data can be obtained by querying the local database and conduct computing locally. We have obtained the performance benchmark of the system from two applications and both have satisfactory results. When it is applied to global climate simulation, the model simulation is carried out on ALPS supercomputer, and the resultant temporal data are distributed to slave data nodes for parallel post-processing by using MPI. The global forecast data can be further downscaled in regional and local areas through different spatial-scale refinement of models or statistical approach of data mining. When applied to air pollution monitoring, the platform is connected to EPA open data which are used as an input for air pollution GTx model simulation. The simulation and data post-processing are both carried out by GTx on the slave nodes by way of distributed and shared-nothing processing. The influence weighting between point source and receptor or local monitor station thus can be determined. Air quality monitoring requires consider all kind of scenarios, including fixed point source and mobile source management. Therefore, it is necessary to run for many combinations of cases and to constitute knowledge base for fast decision support. Further study will be focused on the air pollution per-warning and response in Taichung city which will be linked with the smart city operation in the city.
\end{abstract}

\section{Categories and Subject Descriptors}

E.1 [DATA STRUCTURES]: Distributed data structures; I.1.4 [Applications]; I.6.6 [Simulation Output Analysis]; I.6.8 [Types of Simulation]: Distributed, Parallel, Visual

\section{General Terms}

Management, Performance, Design, Economics, Experimentation, Human Factors

\section{Keywords}

In-Place Computing, Big Data Platform, Parallel Visualization, Distributed Database, Data Node, Air Pollution, Climate Model.

\section{Introduction}

High-performance computing enables scientists using numerical modeling to explore natural phenomena and resolve engineering design problems. In accordance with the progress of computing power and storage capacity in computer, we are able to predict physical phenomena and solve complicate, multi-scale problem more accurately and look deep insight into the unknowns. However, due to the growth of computing scale and physical complexity, the data size produced from output of numerical simulation appears to have increased explosively. The data grow from Gigabyte $\left(10^{9}\right)$, Terabyte $\left(10^{12}\right)$ to above Petabyte $\left(10^{15}\right)$. It creates great impact on the data storage capacity, as well as the capability to analyze such huge size of data. As a consequence, high-performance computing, which usually accompanies with Terabytes data produced by model simulation, has created new challenges in data storage management, transmission, and analysis. Under these circumstances, the problem is to build a system which meets the requirement of performing large-scale model simulation and huge data management and analyses. Therefore, the HPC-based big data platform is a good solution to resolve this type of problem [1]. 
In general, numerical simulation through high-performance computing is to use domain decomposition method to partition the computational domain, and the data in each domain are sent to computing node for computation. The outcome of computation then is send back and store in the data storage. This is traditional way data bundled by computing node. However, it is different story the way of big data management and analysis that the data are distributed to local data node for designate processing, for example, the data server and processing procedure utilized in the parallel visualization software ParaView [2] and VisIt [3]. It is because it is more efficient to deal with data in data node than that of migrating data to computing node.

Regarding the climate and environmental related applications, the output data produced by simulation model have the feature of grid-field structure [4]. That means the data corresponding spatial coordinates are coincident with those of computational grids. It is structured data type. If we consider the programming adaptability of numerical model, Hadoop streaming is able to convert the existing numerical program into JAVA code. However, it is tedious to formulate Map-Reduce programming if the existing model is too big. Therefore, Hadoop [5] which is unstructured database, like NoSQL, may not be ready immediately applied for climate and environmental problems. Actually, using the structured database SQL is sufficient for those applications. Moreover, streaming processing for big data is a must for climate forecast and environmental monitoring applications, such as visualization. Applying parallel processing for big data is a challenging task. It may happen that data size can be over the hard memory limit. However, some useful methods, such as In-Situ visualization [6] or Query Driven Visualization (QDV) [7, 8, 9] can be used in this situation. In-Situ visualization implements visualization function into simulation model. The simulation produces visualization straight forward and thus saves the storage of output data. The disadvantage is it requires re-running model simulation if there is slight change in the visualization plan. For QDV method, it is to analyze existing data set. In the data node, it only reads relevant data through the query of database.

The current big data platform adopted concepts of QDV and Hadoop's in-place computing [10], using local distributed database and distributing the huge data to local data node for parallel post-processing/visualization. The benchmarks are implemented for both global climate simulation and air pollution monitoring applications.

\section{System Architecture}

\subsection{Hardware}

The big data platform is designed for experiments on the climate model and air quality monitoring applications. Therefore the hardware capacity at this moment is to integrate the currently available resources. Data node capacity will be upgraded after the proposed application benchmark tests are accomplished and satisfactory. Currently, the platform is comprised of four components, Advanced Large-scale Parallel Supercluster (ALPS) [11], storage pool, one master data node and 18 slave nodes, as shown in Figure 1. The ALPS supercomputer is responsible for global climate model simulation and the output is stored in the storage pool which can hold 160TB of data. The master node is the coordinator among ALPS, storage pool and 18 slave nodes. The network between ALPS and master node is 10 GE (Giga bit per second Ethernet), the others are 1GE. In the air pollution monitoring application, model simulation and data analysis both will be carried out on slave nodes.

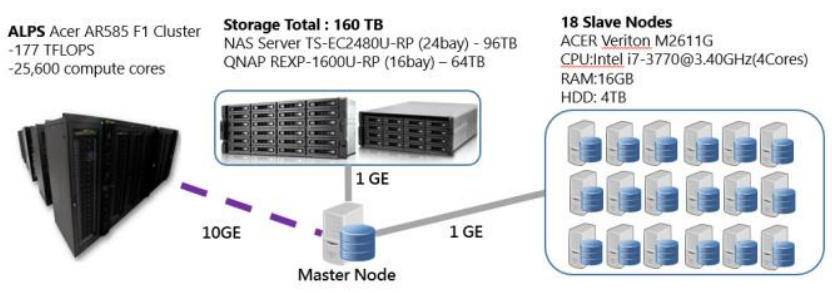

Figure 1. System hardware architecture of the big data platform.

\subsection{Software and Methodology}

The openSUSE operating system [12] and MaraiaDB database [13] are installed on the master node and 18 slave nodes. The core concept of big data analysis is in-place computing. The data are the key for in-place computing. Application programs will be executed at the place where data are located. That means data are distributed and stored on hard drives of slave data nodes through the distributed file system. Then the application program is assigned to the location of the data node to read local data and perform analysis. Each node has its own data database. When data analysis program carries out its application on the data node, it simply refers to the local database where data are situated. However, before the previous mentioned processes, the whole data set should be decomposed into blocks and allocated to database of each data node. Currently the data were partitioned to blocks according to the timestamp attributes of data; and the round robin algorithm is used for data partition to sequence of slave nodes.

\section{Application and Benchmark}

Two real world applications, parallel post-processing of global climate simulation data and air pollution monitoring, are selected as use cases and for performance benchmark test. Figure 2 shows the framework of two applications on the big data platform. The simulation is executed on ALPS supercomputer and postprocessing is conducted simultaneously on the data nodes such as the climate simulation case, or carry out both model simulation and parallel visualization in data nodes such as the air pollution monitoring case.

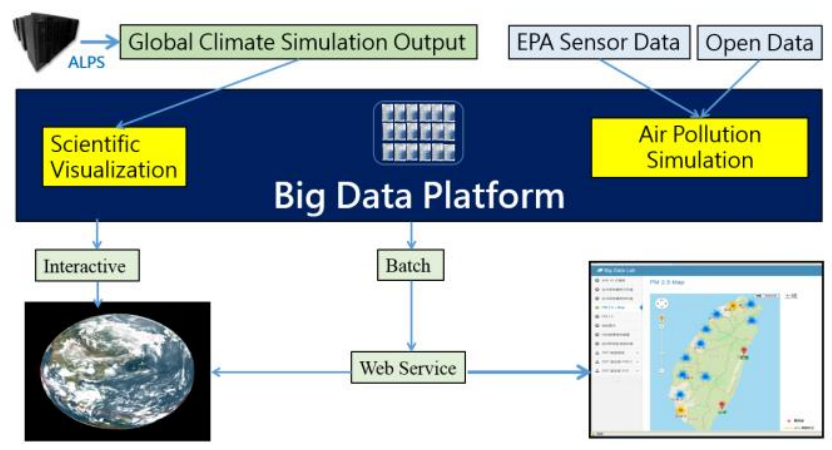

Figure 2. Application framework in the big data platform.

\subsection{Parallel Post-processing of Global Climate Data}

The first use case of the platform application is parallel postprocessing for the visualization of global climate model simulation [14, 15]. The accumulated data size of historic temporary and spatial data from the global climate model simulation output has more than 200TB. Here, we only select 
limited cases for demonstration. The output data of model is in netCDF [16] format which is popularly used in the atmospheric science. The output data size in space is $1536 \times 768$, and 248 time steps. The attributes of this simulation include Outgoing Longwave Radiation (OLR), precipitation, temperature, wind field, and others. The whole dataset can be decomposed into local nodes based on spatial or temporal partition. In this case study, temporal decomposition is adopted. For example, the output of climate model simulation produces 248 time series of data sets in NetCDF format which can be treated as 248 blocks, these blocks were in turn parallel allocated to local database on each data node, as shown in Figure 3; while the database on master server node stores metadata which contains the information of data decomposition status (such as which slave node the data block is stored, attribute names, dimensions, etc.) and integrate analysis result accomplished in each slave data node.

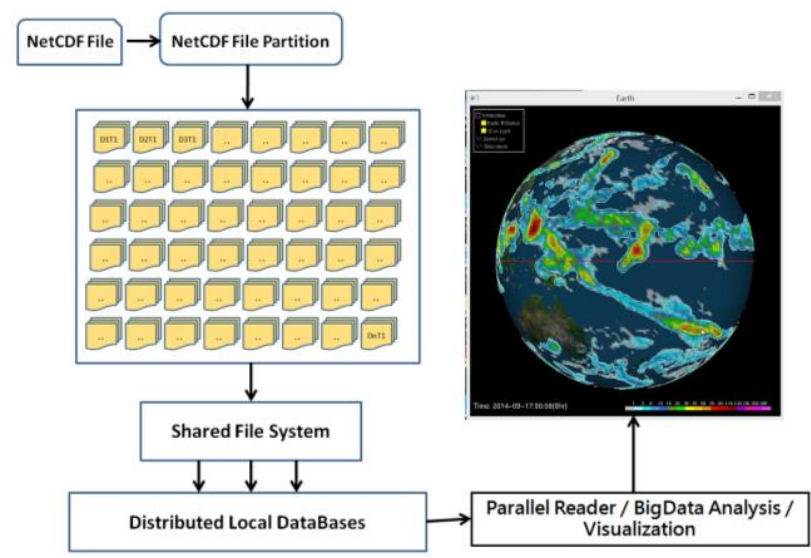

Figure 3. NetCDF file partition for parallel visualization.

Figure 4 shows the detailed processing workflow for the postprocessing task. We implement a client-server frame work to allow the user communicates with the master server node. User client proposes an analysis demand to server. Based on the demand, server node retrieves metadata from its database and distributes the information to slave nodes. The round robin algorithm is used for data partition to sequence of slave nodes. Consequently, slave data node is able to know what data are required with the metadata from mater node, and retrieve data by querying local database, then perform needed analysis/computation. Because the data is partitioned according timestamp, each dataset is independent to the others, the only communications needed are between master and slave nodes carried out by MPI. The analysis/computation results in local node are then sent back to master server node. After master node collects computation results from the slave nodes, the visualization procedure can be carried out on master node and send result images back to user, or user retrieves all the computation results and visualizes the result on his machine.

Under the framework, the data nodes may need necessary communication with each other. For practical visualization of OLR attributes of the simulation results, which are stored in the slave data nodes. Each temporal OLR data set is analyzed/computed on slave node to create contour lines information which is collected and integrated by the server or master node. Finally, the server node can display each temporal image, as seen in Figure 5, as well as the time sequential dynamic animation. Since totally there are 248 time steps of data that are evenly distributed to 17 slave nodes. Each slave node is responsible for processing around 14 15 data sets.

Overall speaking, this approach can be applied not only for post processing of OLR data, but also for other output variables such as temperature, rainfall, and et al. The selected post processing images of temperature, rainfall are shown in Figures 6 and 7, respectively. The performance benchmark is carried out using the 17 slave data nodes for parallel analyses of OLR data set, and we found that it takes 670 seconds to compute contour information of all 248 data sets sequentially in one machine, and it only takes 58 seconds using our distributed parallel system. It is worth to know the current network interconnection is just $1 \mathrm{GE}$ between master node and slave nodes, because current status is for experiment. The performance can be even better if the interconnection is upgraded. This work demonstrates that we are able to increase the performance by the proposed big data platform while using the output data sets produced by global climate model simulation. However, the performance benchmark only measures the computation time, it doesn't count the time to partition and distribute blocks to local slave nodes which will carry out once when data arrived.

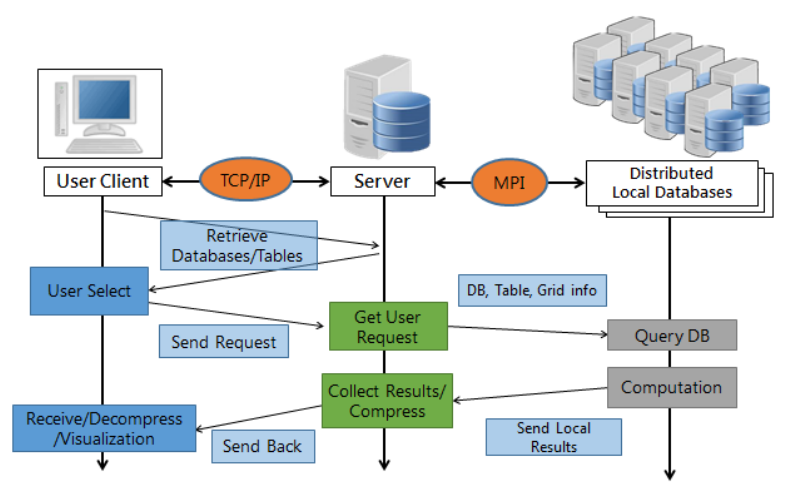

Figure 4. Detailed processing flow for visualization task: Client sends a request to server. The server plays another role of master data node. Master data node can communicate with slave data nodes for data distribution and subsequent data visualization, and collect analyzed information accomplished by slave nodes. The server then integrates analyzed information of various time domains or space domains for a complete data presentation.
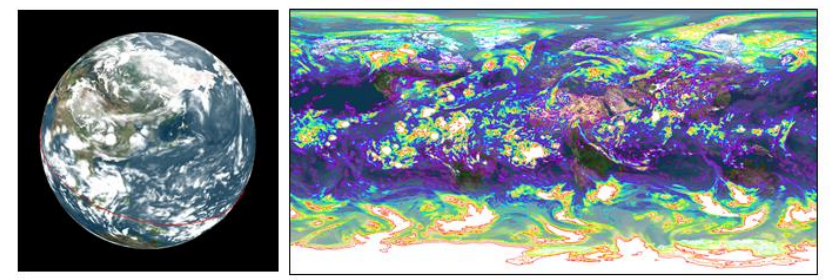

Figure 5. Visualization of OLR output data: Left-hand one shows that white color OLR represents strong vertical convection, and near transparent color indicates high-level cloud with less precipitation chance. Right-hand figure is to display in regular color table. The display background can be $2 \mathrm{D}$ plane or $3 \mathrm{D}$ sphere/earth.

It is worth to mention above that Tsuang used EHTW global climate model [14] for 45-days Forecast. The global forecast data can be downscaled to regional and local areas through different 
spatial-scale refinement of models or statistical approach of data mining, such as nonparametric statistical downscaling. For example, the temperature data as shown in Figure 6 which are of $125 \mathrm{KM}$ resolution in global scale. Therefore, there are only 4 grid nodes falling in Taiwan area. However, it is still possible using nonparametric statistical downscaling method together with long-term observed data to interpolate spatial temperature in anywhere of Taiwan with less one degree of error.

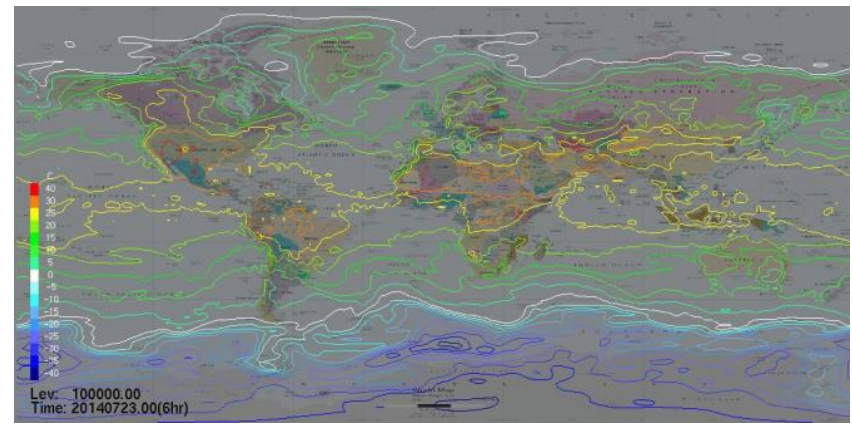

Figure 6. Visualization of output data: selected image of global temperature distribution. The display background is in $2 \mathrm{D}$ plane.

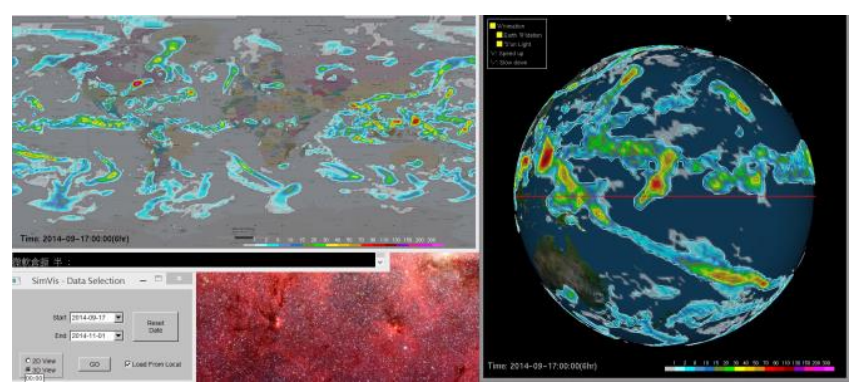

Figure 7. Parallel visualization of output data: selected images of global rainfall distribution. The display background can be $2 \mathrm{D}$ plane or 3D sphere/earth.

\subsection{Air Pollution Simulation Model}

In this study, Taiwan Environmental Protection Agency (EPA) air quality open data is used as an input to run the air pollution simulation model to find out the contribution relationship from primary pollutants (point sources) to EPA monitor stations. The model simulation and data visualization are both carried out in the slave nodes in way of distributed and parallel processing. The influence weighting between point source and receptor or local monitor station can be mined through series of runs that supports the pre-warning and response of air quality in a city.

Currently, EPA Taiwan has maintained 78 air quality monitor stations, each station provides pollutant standards index (PSI), $\mathrm{SO} 2, \mathrm{CO}, \mathrm{O} 3, \mathrm{PM} 10, \mathrm{PM} 2.5, \mathrm{O} 3, \mathrm{NO} 2$, wind speed, wind direction and floating particulate matter monitor data which is updated every hour and published on EPA open data website to coordinate with the open governmental information policy. In addition to EPA open data, Taiwan Central Weather Bureau (CWB) surface weather map which is updated every day is also used to support for understanding every day's weather condition.

The air pollution simulation model we selected is based on a Gaussian plume trajectory model (GTx) and improved by Taiwan academia researchers $[17,18]$. The advantage of GTx is that the source/receptor relationship of sources can be determined in a single model run. The fraction of point source affecting receptor can be simulated in each model run. This is defined as forward trajectory simulation. On the contrary, based on the receptor, the fraction contributed by various source to receptor can also be evaluated through model simulation, and it is backward trajectory simulation. Overall speaking it needs to run many combination pairs of each source and each receptor in area of interest.

\section{Air Pollution Monitoring}

The air pollution simulation framework is shown in Figure 8. We write a program to read and parse EPA open data through web service and then write EPA data to master database. Once master database is updated, all slave databases will be updated automatically by database automatic replication mechanism. The database is on incremental replication to all data nodes, it means that only newly added records are replicated to slave nodes. The round robin algorithm is used for data partition to sequence of slave nodes. It is shared-nothing storage scheme, and therefore, there is no communication among salve nodes. Communication only exists between server node and slave node.

The GTx simulation model introduced above is installed on each node and EPA monitor data is replicated to each computation node. Therefore, the GTx simulation model can read local data (i.e., in-place computation) to execute simulation without taking time and effort to read input data from other nodes. This is so named in-place computation and its advantage is that it saves time of moving huge data.

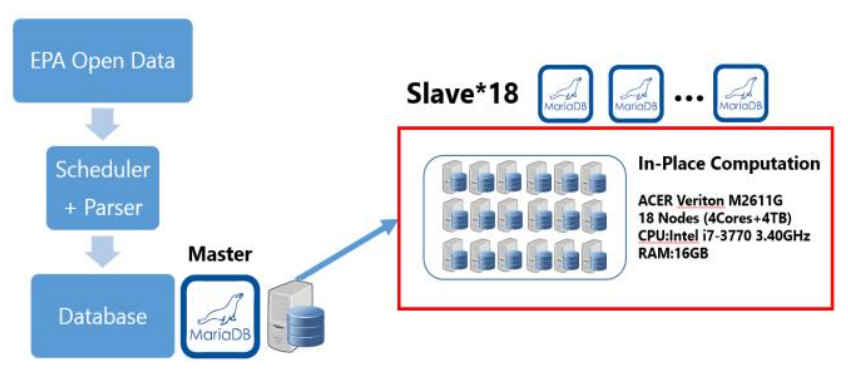

Figure 8. In-place computation framework for air pollution monitoring: model simulation, and data visualization and analysis are carried out in the slave data nodes.

\subsection{Simulation Workflow and Performance Benchmark}

Figure 9 shows GTx simulation model's workflow. The model simulation is to predict for the next day but using observation data in the past two days for kind of data assimilation [18]. EPA monitor station data is stored in MariaDB, the "traj" subroutine reads EPA monitor stations data as input to setup initial condition, the output is stored in local hard disk, then the GTx simulation is launched which reads traj's output as input for computation, GTx's output is also stored in local hard disk for "grads" subroutine to draw output graph.

At first time, we repeat the workflow on master node 73 times for 73 EPA monitor stations, it takes 9 hours.

At second time, we use 17 available slave nodes (each node has 4 cores and 8 threads) to solve the same problem concurrently with mounted shared file system at master node, it takes 50 minutes. 
At third time, we use 17 slave nodes to solve the same problem concurrently with local database and file system, it takes only 10 minutes.

The reason why we used 17 slave nodes instead of 18 slave nodes we mentioned in Figure 8 is because 1 slave node was malfunction at that time.

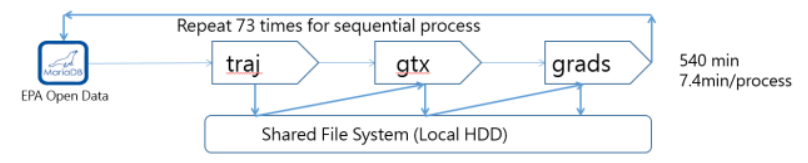

Distributed process: 73 cores, 17 nodes

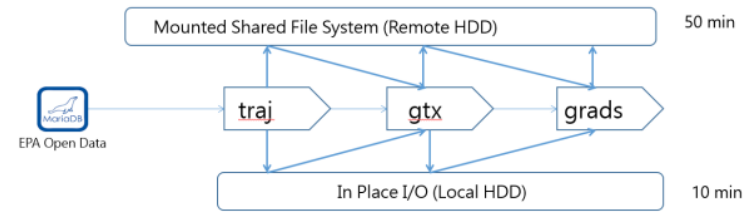

Figure 9. Workflow of air pollution model GTx simulation and performance benchmark: For 3-day air pollution model simulation using slave nodes only takes 10 minutes of computation time; while the sequential run takes 9 hours of computation time.

\subsection{User Interface}

We have built a website to show the output of the two applications running on big data platform. In this section, we show some screen shots of the user interface related to air pollution simulation.

\subsection{EPA Monitor Stations' Monitoring Data}

As shown in Figure 10, Google map on the left hand side is used to show the geolocation of EPA monitor stations. Each monitor station on Google map is a hyperlink. When user selects a monitor station, the selected monitor station's IP cam image and every hour's monitoring air quality data are displayed.
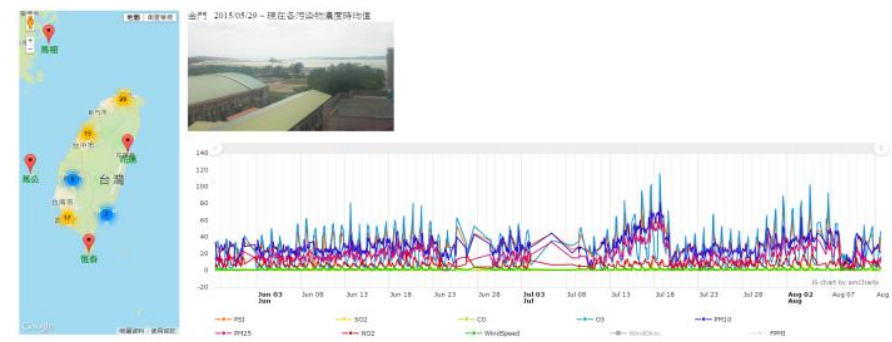

Figure 10. EPA monitor station: geolocation and hourly air quality data.

\subsection{GTx Air Pollution Simulation Results}

The air pollution simulation model GTx is executed once a day. Therefore, user can select one day in the past to see that day's observed air quality and simulation result. Figure 11 shows the user interface for user to select one day of surface weather map in the past. Figure 12 shows the selected day's surface weather map, observed air quality and GTx simulation results.

Since there are many pollutants and many EPA monitor stations, there are too many possible combinations. Here, only the most important pollutant PM2.5 is selected as an example to illustrate.

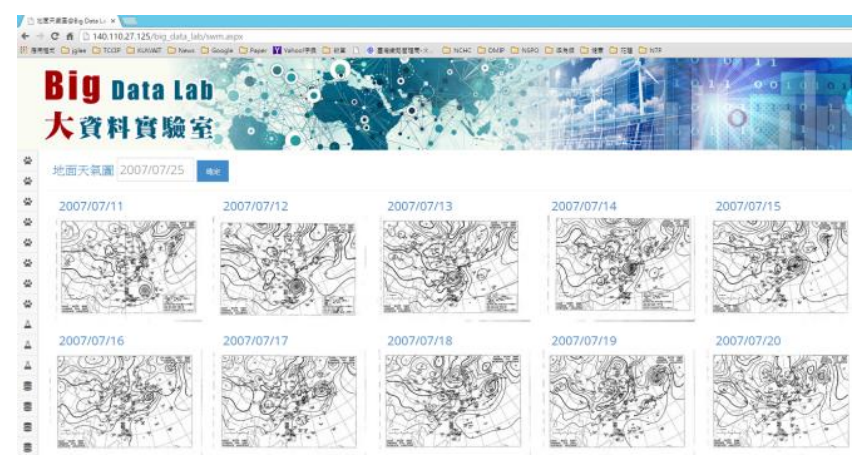

Figure 11. Central Weather Bureau surface weather map.

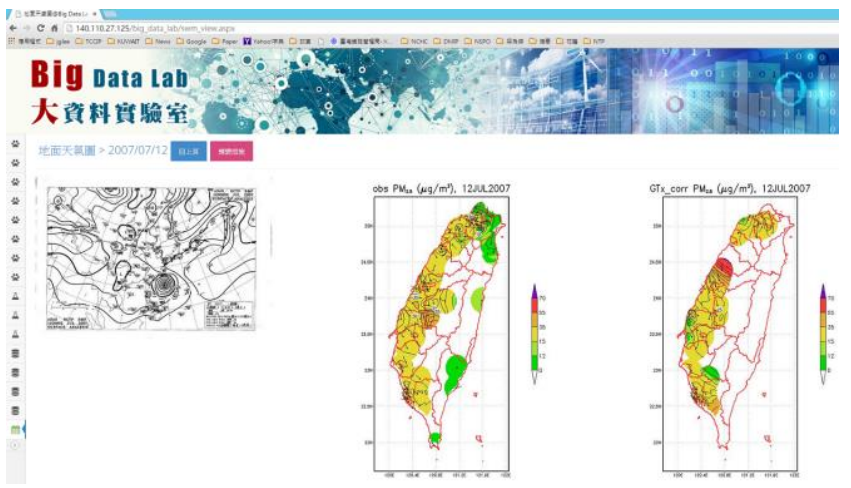

Figure 12. PM 2.5 geographic distribution in Taiwan: comparison between observation and simulation results.

After selecting a day in the past, we can see that day's surface weather map, observed air quality and simulation result. User can see more detailed information as shown in Figure 13. Google map on the left-hand side shows the geolocation of EPA monitor stations, user can select one monitor station; the right-hand side shows the contributed concentration of each point source pollutant to the monitor station. User can even try to change the contributed concentration of pollutant in each point source. The system will then run the simulation again and show the new simulation result. The influence weighting between point source and receptor or local monitor station can be identified through series of runs that will support the pre-warning and response of air quality in a city.

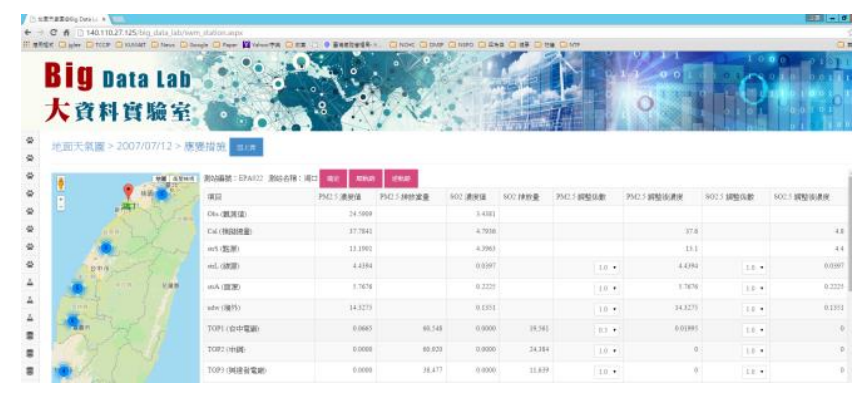

Figure 13. A list of various analyzed PM 2.5 concentration at monitor station, which are induced from various point sources. It is convenient to monitor the air pollution by constructing the influenced weighting on the receptor caused by all point sources. 


\section{Concluding Remarks}

Climate forecast and air pollution monitoring require a system that is capable of dealing both large-scale simulation and big data analytics. In the proposed experimental big data platform, it accommodates the spirits of HPC distributed computing and Hadoop's in-place computing. Master and slave distributed database is implemented in the data nodes. The database MariaDB is set up at both 18 slave cluster nodes which are responsible for data storage and distributed parallel computing, and master data node for managing metadata and further processing the information obtained from cluster nodes. The metadata includes the information of data, such as attributes, the partition table.

The benchmark performance has been carried out by using global climate simulation resultant data with satisfactory results. The platform, connected to governmental open data, then is used for air pollution model simulation and data analytics for pre-warning and response of air quality study. For the 3-day air pollution model simulation using slave nodes only takes 10 minutes of computation time; while the sequential run will take 9 hours of computation time. It is found the platform plays a good role on inplace computing, and the parallel visualization delivers temporal and spatial air quality forecast result effectively, that provide decision alternatives associate with reduction of point source management. The workflow from model simulation, data visualization, and web service has been integrated for automate execution and display.

The global forecast data can be downscaled to regional and local areas through different spatial-scale refinement of models or statistical approach of data mining, such as nonparametric statistical downscaling. It is possible using nonparametric statistical downscaling method together with long-term observed data to interpolate spatial temperature in anywhere of Taiwan with less one degree of error. Forecast data, such as temperature and rainfall prediction, after downscaled to local areas can add the value and become the business service products.

Air quality monitoring requires consider all kind of scenarios, including fixed point source and mobile source management. Therefore, it is necessary to run for many combinations of cases and to constitute knowledge base for fast decision support. Further study will be focused on the air pollution per-warning and response in Taichung city which will be linked with the smart city operation.

Regarding the climate and environmental related applications, the output data produced by model simulation have the feature of grid-field structure, and is structured data type. Many science and engineering applications have similar data structure, and can be potential use cases for the current developed big data platform. Also, the capacity of the big data platform will be continued to upgrade to meet demands of various similar scientific and engineering applications.

\section{ACKNOWLEDGMENTS}

The authors would like to express sincere appreciation to EPA and Central Weather Bureau, Taiwan, for providing weather and air quality observation data.

\section{ADDITIONAL AUTHORS}

I-En Liao (National Chung-Hsing University, Taiwan, Email: ieliao@nchu.edu.tw); Chia-Ying Tu, Academia Sinica, Taiwan,
Email: cytu@gate.sinica.edu.tw); Kai Chen Ku (National ChungHsing University, Taiwan, Email: dodo1654.ku@gmail.com); Shinyii Liu (National Chung-Hsing University, Taiwan, Email: shinyii.liu@gmail.com,); T.-M. Chuang (National Center for High-performance Computing, NARL, Taiwan, Email: tmchuang@nchc.narl.org.tw); Wen-How Cheng (National ChungHsing University, Taiwan, Email: tom177832@gmail.com)

\section{REFERENCES}

[1] Lee, L.-C., Tsai, W.-F., Lin, S.-C., Hsu, W.-C. 2014. Big Data Post Processing for Active Magnetic Regenerator Thermal Flow Simulation. Paper No. 27-17, The 21th Taiwan CFD conference, Nantou, Taiwan.

[2] ParaView software. http://www.paraview.org/

[3] VisIt software. https://wci.llnl.gov/codes/visit/

[4] Howe, B. and Maier, D. 2004. Algebraic Manipulation of Scientific Datasets. Proceedings of the 30th VLDB Conference,Toronto, Canada.

[5] Hadoop: http://hadoop.apache.org/

[6] Ma, K. L. 2009. In Situ Visualization at Extreme Scale: Challenges and Opportunities Computer Graphics and Applications. IEEE vol.29, pp.14-19.

[7] Stockinger, K., Shalf, J., Wu, K., and Bethel, E. W. 2005. Query-Driven Visualization of Large Data Sets. Visualization Conference, IEEE, 16th IEEE Visualization, $p$ 22.

[8] Gosink, L. J., Anderson, J. C., Bethel, E. W., and Joy, K. I. 2008. Query-Driven Visualization of Time-Varying Adaptive Mesh Refinement Data. IEEE Transactions on Visualization and Computer Graphics, vol. 14, pp. 1715-1722.

[9] Zou, H. B., Slawinska, M., Schwan, K., Wolf, M., Eisenhauer, G., Zheng, F., Dayal, J., Logan, J., Klasky S., Bode, T., Kinsey, M., and Clark, M. 2013. FlexQuery: An Online Query System for Interactive Remote Visual Data Exploration at Large Scale. IEEE Cluster 2013, Indiannapolis, IN.

[10] Dean, J. and Ghemawat, S. 2004. MapReduce: Simplified Data Processing on Large Clusters. OSDI'04: Sixth Symposium on Operating System Design and Implementation, San Francisco, CA..

[11] Advanced Large-scale Parallel Supercluster (ALPS), http://www.nchc.org.tw/en/

[12] OpenSUSE: https://www.opensuse.org

[13] MariaDB: https://mariadb.com/

[14] Tu, C.-Y. and Tsuang, B. J. 2005. Cool-skin simulation by a one-column ocean model. Geophysical research letters, 32(22), L22602.

[15] Tsuang, B. J., Tsai, J. L., Lin, M. D., and Chen, C. L. 2003. Determining aerodynamic roughness using tethersonde and heat flux measurements in an urban area over a complex terrain. Atmospheric Environment, 37(14), 1993-2003.

[16] NetCDF: http://www.unidata.ucar.edu/software/netcdf/

[17] Tsuang, B. J. 2003. Quantification on the source/receptor relationship of primary pollutants and secondary aerosols by a Gaussian plume trajectory model: Part I-theory. Atmospheric Environment 37 (28), 3981-3991. 
[18] Tsuang, B.J., Chen, C.L., Lin, C.H., Cheng, M.T., Tsai, Y.I., Chio, C.P., Pan, R.C., Kuo, P.H. 2003. Quantification on the source/receptor relationship of primary pollutants and secondary aerosols by a Gaussian plume trajectory model:

Part II. Case study. Atmospheric Environment, 37(28), 3993-4006. 